\title{
BMJ Open Finnish National Esophago-Gastric Cancer Cohort (FINEGO) for studying outcomes after oesophageal and gastric cancer surgery: a protocol for a retrospective, population-based, nationwide cohort study in Finland
}

\section{Joonas H Kauppila, ${ }^{1,2}$ Pasi Ohtonen, ${ }^{3}$ Tuomo J Karttunen, ${ }^{4}$ Arto Kokkola, ${ }^{5}$ Simo Laine, ${ }^{6}$ Tuomo Rantanen, ${ }^{7}$ Ari Ristimäki, ${ }^{8}$ Jari V Räsänen, ${ }^{9}$ Juha Saarnio, ${ }^{3}$ Eero Sihvo, ${ }^{10}$ Vesa Toikkanen, ${ }^{11}$ Tuula Tyrväinen ${ }^{12}$}

To cite: Kauppila JH, Ohtonen $\mathrm{P}$ Karttunen TJ, et al. Finnish National Esophago-Gastric Cancer Cohort (FINEGO) for studying outcomes after oesophageal and gastric cancer surgery: a protocol for a retrospective, populationbased, nationwide cohort study in Finland. BMJ Open 2019;9:e024094. doi:10.1136/ bmjopen-2018-024094

- Prepublication history and additional material for this paper are available online. To view these files, please visit the journal online (http://dx.doi. org/10.1136/bmjopen-2018024094).

Received 9 May 2018 Revised 10 0ctober 2018 Accepted 16 November 2018

Check for updates

(C) Author(s) (or their employer(s)) 2019. Re-use permitted under CC BY-NC. No commercial re-use. See rights and permissions. Published by BMJ.

For numbered affiliations see end of article.

Correspondence to Dr Joonas H Kauppila; joonas.kauppila@oulu.fi

\section{ABSTRACT}

Introduction Surgery for oesophageal and gastric cancers is associated with high morbidity, mortality and poor quality of life postoperatively. The Finnish National Esophago-Gastric Cancer Cohort has been established with the aim of identifying factors that could contribute to improved outcomes in oesophago-gastric cancer.

Methods and analysis All patients with oesophageal and gastric cancer diagnosed in Finland between 1987 and 2015 will be identified from the Finnish national registries. The Finnish Cancer Registry and Finnish Patient Registry will be used to identify patients that fulfil the inclusion criteria for the study: (1) diagnosis of oesophageal, gastrooesophageal junction, or gastric cancer, (2) any surgical treatment for the diagnosed cancer and (3) age of 18 or over at the time of diagnosis. Clinical variables and complication information will be retrieved in extensive data collection from the medical records of the relevant Finnish hospitals and complete follow-up for vital status from Statistics Finland. Primary endpoint is overall all-cause mortality and secondary endpoints include complications, reoperations, medication use and sick leaves. Sub-studies will be implemented within the cohort to investigate specific populations undergoing oesophageal and gastric cancer surgery. The initial estimated sample size is 1800 patients with surgically treated oesophageal cancer and 7500 patients with surgically treated gastric cancer. Ethics and dissemination The study has been approved by the Ethical Committee in Northern Ostrobothnia, Finland and The National Institute for Health and Welfare, Finland. Study findings will be disseminated via presentations at conferences and publications in peer-reviewed journals.

\section{INTRODUCTION}

Gastric cancer is the third, and oesophageal cancer the sixth, leading cause of cancer death worldwide. ${ }^{1}$ The incidence of oesophageal adenocarcinoma is increasing, while
Strengths and limitations of this study

- The main strength of the study is the population-based design with complete and accurate ascertainment and follow-up of all patients diagnosed with oesophageal or gastric cancer in Finland, counteracting selection bias.

- The inclusion of multiple potential confounding factors from the registries and patient records allows accurate analysis of a variety of exposures and end points.

- The sample size will large enough to enable robust survival and regression analyses in smaller subgroups of patients.

- The main limitations of the study are the exclusion of patients not undergoing surgery and information lag of up to 2 years.

that of oesophageal squamous cell carcinoma and gastric cancer is decreasing in Finland, ${ }^{2}$ similar to other Western countries. ${ }^{3}$ The incidence of gastric cancer is slowly decreasing in majority of the countries across the globe. ${ }^{4}$ However, both cancers are characterised by poor survival even after curatively intended surgery $^{5-8}$ and a 'textbook outcome' may be achieved in less than half of the patients undergoing oesophago-gastric cancer surgery. ${ }^{9}$ Studies on sick leaves, ${ }^{10}$ or postoperative use of opioids as an outcome after oesophageal and gastric cancer surgery are lacking, while these are important outcomes for the patients. It has been shown that sick leaves affect for example job retention in patients with cancer. ${ }^{11}$

Randomised trials in oesophageal and gastric cancer have provided quality 
evidence that neoadjuvant-and adjuvant therapies increase survival ${ }^{12-15}$ and that minimally invasive surgical approaches reduce, or at least do not increase complications. ${ }^{16}{ }^{17}$ Despite their good internal validity and lack of bias, randomised controlled studies in general have limited external validity and applicability to general population, and thus need to be complemented by quality observational studies to reliably assess the effects of implementation of trial results into practice. ${ }^{18}$ Additionally, observational studies can provide evidence on questions that have not, or cannot, be evaluated in randomised trials. $^{18}$

In Finland, high-quality registry data on these cancers is readily available. ${ }^{19}$ Despite the good availability, the exposure and outcome data in the registries are not detailed enough for surgical research. Because of small population ( 5.5 million) sparsely populating Finland, the hospitals are many, and the single-centre cohorts containing detailed information are small, not necessarily generalisable and have potential selection bias. There are no previous coordinated nationwide population-based research efforts with detailed clinical data on oesophago-gastric cancer in Finland. To overcome these challenges, an extensive retrospective nationwide data collection from the patient medical records is needed. The Finnish National Esophago-Gastric Cancer Cohort (FINEGO) was established as a researcher-led effort to coordinate this retrospective database.

\section{Objectives}

The specific objectives of the FINEGO are:

- To establish important baseline data on national and regional trends and changes over time in oesophageal and gastric cancer surgery, postoperative morbidity and long-term outcomes in Finland.

- To investigate associations between surgeon and hospital volume and postoperative morbidity and mortality in oesophago-gastric cancers.

- To assess the relevance of clinical characteristics, modifiable risk factors, such as preoperative feeding, surgical approach, type of neoadjuvant treatment, or method of analgesia in relation to mortality and morbidity outcomes, as well as cancer survivorship, such as postoperative medication use in oesophago-gastric cancer.

- To investigate whether histological assessment could be used for prediction of prognosis in oesophageal and gastric cancer.

\section{METHODS}

A multicentre FINEGO-collaborative has been established to conduct large-scale epidemiological, clinical and clinicopathological studies in oesophago-gastric cancers. The collaborative includes one to two senior consultants upper gastrointestinal or thoracic surgeons as the local principal investigators (PIs) from all academic centres conducting oesophageal and gastric cancer surgery in Finland, as well as senior consultant oesophago-gastric pathologists and biostatisticians. The participating researchers will sign the needed professional confidentiality consents to be allowed access to patient data obtained from the registries.

\section{Study design}

This study is a population-based, nationwide, retrospective cohort study in Finland. The initial study period is from 1 January 1987 to 31 December 2015, with follow-up until 31 December 2016. The study period will be expanded every 5 years to keep the cohort updated for the most recent data.

\section{Inclusion and exclusion criteria}

The patients fulfilling the following inclusion criteria are included in the study:

1. Primary cancer of epithelial origin in the oesophagus, the gastro-oesophageal junction or the stomach.

2. Patient receives surgical treatment for the cancer, including curative, palliative, rescue surgery that is, surgery after curative chemoradiation, or endoscopic curative surgery, such as endoscopic mucosal resection and endoscopic submucosal dissection.

3. Age at least or over 18 years during the time of diagnosis.

\section{Identification of the study participants}

The immutable, 11-digit personal identification number assigned to each resident in the country allows reliable identification and combining the registry data with patient records. ${ }^{20}$ The patients will be identified through the Finnish Cancer Registry, and the Finnish Patient Registry by searching these registries for cancer diagnoses (online supplementary table 1) and operation status and operations codes (online supplementary table 2 ). The identification through both registries is done to ensure near $100 \%$ completeness on oesophago-gastric cancer diagnosis. The patients undergoing oesophageal or gastric cancer surgery will be identified using the operations codes in the Finnish Patient Registry. The Finnish Patient Registry will provide the hospital names and operation dates, based on which the relevant patient records will be retrieved from the archives of the hospitals in all 21 hospital districts in Finland.

\section{Data collection}

Registry data will be collected from The Finnish Cancer Registry, The Finnish Patient Registry, The Population Register Centre, Statistics Finland and The Social Insurance Institution (Kela)-registry (table 1). The quality of data in these registries is known to be very high ${ }^{21-24}$ and reporting to the registries is mandatory by the Finnish Law. These registry data include the identifying information, the variables related to the socio-economy, and will be used to calculate the well-validated Charlson's Comorbidity Index (online supplementary table 3$)^{25}$ and annual hospital volumes. All registry data-derived variables are calculated by a biostatistician. The patient records of the included patients identified from the registries will be scrutinised by 
Table 1 Data sources and dataset information

\begin{tabular}{ll}
\hline Data source & Variables \\
\hline $\begin{array}{l}\text { The Finnish Cancer } \\
\text { Registry }\end{array}$ & $\begin{array}{l}\text { Personal identification number } \\
\text { (age, sex) } \\
\end{array}$ \\
& Diagnosis number \\
& Date of cancer diagnosis \\
& Tumour stage \\
The Finnish Patient & Personal identification number \\
Registry & (age, sex) \\
& Hospital admissions data \\
& Admitting hospital \\
& Dates of admission and \\
& discharge \\
& Diagnosis codes \\
& Operations codes
\end{tabular}

\begin{tabular}{|c|c|}
\hline $\begin{array}{l}\text { The Population Register } \\
\text { Centre }\end{array}$ & Marital status \\
\hline Statistics Finland & $\begin{array}{l}\text { Education level } \\
\text { Date of death } \\
\text { Causes of death }\end{array}$ \\
\hline $\begin{array}{l}\text { The Social Insurance } \\
\text { Institution (Kela) registry }\end{array}$ & $\begin{array}{l}\text { Dispensed drugs } \\
\text { Type (Anatomical Therapeutic } \\
\text { Chemical (ATC)-code) } \\
\text { Date dispensed } \\
\text { Amount of dispensed drug } \\
\text { Sick leave (start date, end date) } \\
\text { Pension information (start date) }\end{array}$ \\
\hline Patient records & $\begin{array}{l}\text { Tumour stage information } \\
\text { Anaesthesia information } \\
\text { Type of anaesthesia } \\
\text { ASA classification* } \\
\text { Surgery information } \\
\text { Type of surgery } \\
\text { Surgeon volume } \\
\text { Bleeding } \\
\text { Operation duration } \\
\text { Complications } \\
\text { According to the } \\
\text { Esophagectomy } \\
\text { Complications Consensus } \\
\text { Group } \\
\text { Clavien-Dindo classification } \\
\text { Oncological treatment } \\
\text { Neoadjuvant and adjuvant } \\
\text { treatment } \\
\text { Treatment modality } \\
\text { Complications } \\
\text { Pathology } \\
\text { Tumour location and stage } \\
\text { Lymph node yield and } \\
\text { resection radicality } \\
\text { Hospital and intensive care unit } \\
\text { stay }\end{array}$ \\
\hline Biobanks & $\begin{array}{l}\text { Scans of original diagnostic } \\
\text { slides }\end{array}$ \\
\hline
\end{tabular}

the study nurses and the investigators using standardised forms for clinical variables, including patient characteristics and surgeon, outcome and complication information (table 1 and online supplementary text 1 ). The clinical variables have been decided on by the investigators in the FINEGO group. Key variables, such as those from the operations charts will be extracted by one of the investigators, while the nurse extracts information not considered prone to errors, such as administrative data and laboratory results. All records and the corresponding data collection forms will be scanned and saved for later use. The histological samples will be collected from the biobanks. These original, prospectively collected diagnostic slides from the preoperative gastroscopy and the surgical specimen will be sought from the biobanks' archives for the study patients. The sample slides are retrieved according to the biobank policies, and scanned and digitised into a picture form for assessment and review of the histological parameters and neoadjuvant treatment response (table 1).

The identifying information of the selected patients from each hospital district will be provided to the respective PIs and administrative personnel for obtaining the patient records data from the healthcare entities' archives. The obtained registry data, medical and health records data, as well as the digitised histological samples, will be entered into the study database and pseudonymised using study identifiers after the completion of the data collection. The hard copies of some of the study data will be kept in a safe deposit on the premises of University of Oulu. The identification variables from the registries will be kept in an encrypted and password-protected file with limited access granted to only the main biostatistician and the PI of the project. The pseudonymised cohort without identifying information is available for the members of the collaborative for sub-studies within the framework specified below.

\section{Data management and analysis plan}

The data management and analyses in this study will be supervised and conducted by an expert biostatistician (PO). After finishing the data collection, a cohort profile will be published. For the cohort profile, number of new yearly cancer cases and the yearly number of operated cancers will be calculated based on the registry data. The baseline characteristics, that is, number of patients in each group of selected variables will be reported in tables. Overall all-cause mortality will be reported for each cancer type based on the life table method $^{26}$ and depicted using Kaplan-Meier curves.

Each of the sub-studies will be planned in the collaborative with a detailed a priori study protocol describing the rationale, aims, hypothesis and statistical analysis plan including appropriate methods, potential confounding and biases for the particular research question, as well as the biostatistician involved in the analysis.

\section{Data quality assessment}

The data quality in the registries will be checked through comparing the collected clinical data against the data, namely type of surgery and tumour stage obtained from the registries. Internal audit, where a random sample 
of the patient records of 50 patients with oesophageal cancer and 50 patients with gastric cancer will be re-reviewed by another investigator, and the differences between the two assessments will be checked against the original data collection. If there are signs of difficulties in the assessment of certain variables or systematic errors, these variables will be audited in more detail. As of now, no external audit is planned, but all study protocols, data files, statistical syntax used to obtain the results and the end-product will be kept for further potential audits.

\section{Sample size}

One of the main strengths is the large sample size of the study. It is estimated that at least 6000 oesophageal and 25000 patients with gastric cancer will be found in screening during the study period. Of these patients, estimated 30\% have been operated for their cancer, yielding estimates of 1800 oesophageal and 7500 patients with gastric cancer for the study.

With 1800 patients with oesophageal cancer, the estimated power would be $>80 \%$ to reliably detect weak associations $(\mathrm{HR}=1.15)$, given an equal distribution of patients in the exposure groups. With 7500 patients with gastric cancer, the power would be $>80$ to detect an association at the level $\mathrm{HR}=1.07$.

\section{Permissions and registration}

Relevant local permissions and registrations are obtained by the collaborative from all the 21 hospital districts, namely the Lapland Hospital district, Länsi-Pohja Hospital District, Kainuun Social and Health Care Joint Authority, The Hospital district of Northern Ostrobothnia, Soite, The Hospital District of South Ostrobothnia, Pirkanmaa Hospital District, Kanta-Häme Hospital District, Vaasa Hospital District, Satakunta Hospital District, Hospital District of Southwest Finland, Ålands hälso- och sjukvård, Joint Authority of the Helsinki and Uusimaa Hospital District, Päijät-Häme Hospital District, Kymenlaakso Social and Health Services Care, South Karelia Social and Health Care District (Eksote), North Karelia Central Hospital and Honkalampi Centre, East Savo Hospital District, South Savo Social and Health Services, Kuopio University Hospital District and The Central Finland Hospital District, as well as the relevant Biobanks, namely Auria Biobank, Helsinki Biobank, Biobank of Eastern Finland, Central Finland Biobank, Northern Finland Biobank Borealis and Finnish Clinical Biobank Tampere.

Individual informed consent will not be sought from the patients whose data are used in this observational study. Obtaining the informed consent has been waived by the Finnish law. The study will be conducted in accordance with the Declaration of Helsinki.

\section{Patient and public involvement}

Patients or public were not involved in the development of the research question and study design or conducting the present study.

\section{DISCUSSION}

Oesophageal and gastric cancers have poor prognosis, even after curative surgery. ${ }^{5} 6$ The present population-based, nationwide retrospective cohort study will provide information on the recent time trends in the treatment of oesophageal and gastric cancer and identify new, and verify previously identified, modifiable factors related to morbidity, mortality and survivorship after oesophageal and gastric cancer surgery.

The strengths of the FINEGO-cohort include its population-based design with complete and accurate ascertainment and follow-up of all patients diagnosed with oesophageal or gastric cancer in Finland, counteracting selection bias. The combined use of registry and patient records data reduces information bias. The inclusion of multiple potential confounding factors from the registries and patient records allows accurate analysis of a variety of exposures and end points. The sample size will large enough to enable robust survival and regression analyses in smaller sub-groups of patients. Compared with some global collaboratives, such as the Worldwide Esophageal Cancer Collaboration ${ }^{27}$ or Esophagectomy Complications Consensus Group, ${ }^{28}$ the FINEGO can contribute to the scientific community by producing results in a real-life setting including all patients operated for oesophageal and gastric cancers in the country, while the collaboratives typically include a sample of patients operated in high-volume centres. Furthermore, the above-mentioned collaboratives include only oesophageal cancer, while the present study includes both oesophageal and gastric cancer.

There are also limitations in the present study. The retrospective study design is potentially weaker in data quality, compared with a prospective study. However, the retrospective design enables obtaining a large number of patients more quickly than a prospective data collection, and the data quality in the registries the cohort is based on is known to be very high, and the manually collected patient records data will be vigorously checked and validated for quality. Furthermore, a national quality registry for these patients is going to be established, and the prospective clinical data collected in that quality registry can be later used in the updates of the FINEGO-cohort to potentially improve the quality of the more recent data. All patients not undergoing surgery will be excluded, because examining the patient records for all the patients would increase the number of involved healthcare entities significantly to over 250, reducing the feasibility of the data collection. Patients undergoing curatively intended or palliative chemotherapy and/or radiotherapy will be excluded, reducing the possibilities to study patients with disseminated disease or not eligible for any type of surgery. The cohort is planned to be updated every 5 years, and there is a lag of up to 2 years, including quality checks and controls, before the registry data is made available for research, preventing the study group from getting the most recent data for analysis even during the cohort updates. However, this lag will 
not reduce the relative sample size considerably and the effect on follow-up in person-years is minimal due to high mortality rates of the cancers. Furthermore, there are some variables, including smoking history and alcohol use, that are not recorded in the registries and cannot be reliably retrieved from the patient records, as they are not routinely recorded by the healthcare personnel in a structured way. However, the data quality and missing data will be meticulously checked before running the sub-studies, and the missing data will be taken into account by using multiple imputation methods in the analyses to reduce bias from missing data.

Taken together, this population-based, nationwide retrospective cohort study will provide new evidence regarding various unanswered questions in oesophageal and gastric cancer surgery by combining epidemiological and clinical data, as well as complement randomised clinical trials by assessing their findings in an unselected population.

\section{Author affiliations}

${ }^{1}$ Cancer and Translational Medicine Research Unit, University of Oulu and Oulu University Hospital, Oulu, Finland

${ }^{2}$ Upper Gastrointestinal Surgery, Department of Molecular Medicine and Surgery, Karolinska Institutet and Karolinska University Hospital, Stockholm, Sweden

${ }^{3}$ Department of Surgery, Oulu University Hospital, Oulu, Finland

${ }^{4}$ Department of Pathology, Oulu University Hospital and University of Oulu, Oulu, Finland

${ }^{5}$ Department of Surgery, University of Helsinki and Helsinki University Hospital, Helsinki, Finland

${ }^{6}$ The Division of Digestive Surgery and Urology, Turku University Hospital, Turku, Finland

${ }^{7}$ Department of Surgery, University of Eastern Finland and Kuopio University Hospital, Kuopio, Finland

${ }^{8}$ Department of Pathology and HUSLAB, University of Helsinki and Helsinki University Hospital, Helsinki, Finland

${ }^{9}$ Department of General Thoracic and Oesophageal Surgery, Heart and Lung Centre, University of Helsinki and Helsinki University Hospital, Helsinki, Finland

${ }^{10}$ Department of Surgery, Central Finland Central Hospital, Jyvaskyla, Finland

${ }^{11}$ Department of Cardiothoracic Surgery, Heart Center, Tampere University Hospital

and University of Tampere, Tampere, Tampere, Finland

${ }^{12}$ Department of Gastroenterology and Alimentary Tract Surgery, Tampere University Hospital, Tampere, Finland

Contributors JHK developed the study idea, concept and design, initiated the collaborative project, developed data collection tools, obtained permissions and funding and drafted the study protocol. PO was involved in the statistical design, made substantial revisions to the protocol and was involved in the original concept, study design and implementation. TJK was involved in design and implementation of the study, and revised the protocol. AK was involved in design and implementation of the study, and revised the protocol. SL was involved in design and implementation of the study, and revised the protocol. TR was involved in design and implementation of the study, and revised the protocol. AR was involved in design and implementation of the study, and revised the protocol. JVR was involved in design and implementation of the study, and revised the protocol. JS was involved in design and implementation of the study, and revised the protocol. ES was involved in design and implementation of the study, and revised the protocol. VT was involved in design and implementation of the study, and revised the protocol. TT was involved in design and implementation of the study, and revised the protocol. JHK is the guarantor.

Funding This work is supported by research grants from the Sigrid Jusélius Foundation (Sigrid Juséliuksen Säätiö), The Finnish Cancer Foundation (Syöpäsäätiö) and Orion Research Foundation (Orionin Tutkimussäätiö). The funding sources have no role in the design and conduct of the study; collection, management, analysis and interpretation of the data; preparation, review, or approval of the manuscript; or decision to submit the study protocol for publication.

Competing interests None declared.

Patient consent for publication Not required.

Ethics approval The Ethical Committee in Northern Osthrobotnia; The National Institute for Health and Welfare; Statistics Finland and the Office of the Data Protection Ombudsman.

Provenance and peer review Not commissioned; externally peer reviewed.

Open access This is an open access article distributed in accordance with the Creative Commons Attribution Non Commercial (CC BY-NC 4.0) license, which permits others to distribute, remix, adapt, build upon this work non-commercially, and license their derivative works on different terms, provided the original work is properly cited, appropriate credit is given, any changes made indicated, and the use is non-commercial. See: http://creativecommons.org/licenses/by-nc/4.0/.

\section{REFERENCES}

1. Fitzmaurice C, Allen C, Barber RM, et al. Global Burden of Disease Cancer Collaboration. Global, Regional, and National Cancer Incidence, Mortality, Years of Life Lost, Years Lived With Disability, and Disability-Adjusted Life-years for 32 Cancer Groups, 1990 to 2015: A Systematic Analysis for the Global Burden of Disease Study. JAMA Oncol 2017;3:524-48.

2. Weiderpass E, Pukkala E. Time trends in socioeconomic differences in incidence rates of cancers of gastro-intestinal tract in Finland. BMC Gastroenterol 2006;6:41.

3. Malhotra GK, Yanala U, Ravipati A, et al. Global trends in esophageal cancer. J Surg Oncol 2017;115:564-79.

4. Luo G, Zhang Y, Guo P, et al. Global patterns and trends in stomach cancer incidence: Age, period and birth cohort analysis. Int $J$ Cancer 2017; 141:1333-44.

5. Lagergren J, Smyth E, Cunningham D, et al. Oesophageal cancer. Lancet 2017;390:2383-96.

6. Van Cutsem E, Sagaert X, Topal B, et al. Gastric cancer. Lancet 2016;388:2654-64.

7. Kauppila JH, Mattsson F, Brusselaers N, et al. Prognosis of oesophageal adenocarcinoma and squamous cell carcinoma following surgery and no surgery in a nationwide Swedish cohort study. BMJ Open 2018;8:e021495.

8. Asplund J, Kauppila JH, Mattsson F, et al. Survival Trends in Gastric Adenocarcinoma: A Population-Based Study in Sweden. Ann Surg Oncol 2018;25:2693-702.

9. van der Kaaij RT, de Rooij MV, van Coevorden F, et al. Using textbook outcome as a measure of quality of care in oesophagogastric cancer surgery. Br J Surg 2018;105:561-9.

10. Endo M, Haruyama Y, Takahashi M, et al. Returning to work after sick leave due to cancer: a 365-day cohort study of Japanese cancer survivors. J Cancer Surviv 2016;10:320-9.

11. Veenstra CM, Regenbogen SE, Hawley ST, et al. Association of Paid Sick Leave With Job Retention and Financial Burden Among Working Patients With Colorectal Cancer. JAMA 2015;314:2688-90.

12. Shapiro J, van Lanschot JJB, Hulshof M, et al. CROSS study group. Neoadjuvant chemoradiotherapy plus surgery versus surgery alone for oesophageal or junctional cancer (CROSS): long-term results of a randomised controlled trial. Lancet Oncol 2015;16:1090-8.

13. Cunningham D, Allum WH, Stenning SP, et al. Perioperative chemotherapy versus surgery alone for resectable gastroesophageal cancer. N Engl J Med 2006;355:11-20.

14. Macdonald JS, Smalley SR, Benedetti J, et al. Chemoradiotherapy after surgery compared with surgery alone for adenocarcinoma of the stomach or gastroesophageal junction. $N$ Engl J Med 2001;345:725-30.

15. Kelsen DP, Winter KA, Gunderson LL, et al. Radiation Therapy Oncology GroupUSA Intergroup. Long-term results of RTOG trial 8911 (USA Intergroup 113): a random assignment trial comparison of chemotherapy followed by surgery compared with surgery alone for esophageal cancer. J Clin Oncol 2007;25:3719-25.

16. Biere SS, van Berge Henegouwen MI, Maas KW, et al. Minimally invasive versus open oesophagectomy for patients with oesophageal cancer: a multicentre, open-label, randomised controlled trial. Lancet 2012;379:1887-92.

17. Kim HH, Hyung WJ, Cho GS, et al. Morbidity and mortality of laparoscopic gastrectomy versus open gastrectomy for gastric cancer: an interim report-a phase III multicenter, prospective, randomized Trial (KLASS Trial). Ann Surg 2010;251:417-20. 
18. Booth CM, Tannock IF. Randomised controlled trials and populationbased observational research: partners in the evolution of medical evidence. Br J Cancer 2014;110:551-5.

19. Maret-Ouda J, Tao W, Wahlin K, et al. Nordic registry-based cohort studies: Possibilities and pitfalls when combining Nordic registry data. Scand J Public Health 2017;45:14-19.

20. Ludvigsson JF, Otterblad-Olausson P, Pettersson BU, et al. The Swedish personal identity number: possibilities and pitfalls in healthcare and medical research. Eur J Epidemiol 2009;24:659-67.

21. Sund R. Quality of the Finnish Hospital Discharge Register: a systematic review. Scand J Public Health 2012;40:505-15.

22. Leinonen MK, Miettinen J, Heikkinen S, et al. Quality measures of the population-based Finnish Cancer Registry indicate sound data quality for solid malignant tumours. Eur J Cancer 2017;77:31-9.

23. Lahti RA, Penttilä A. The validity of death certificates: routine validation of death certification and its effects on mortality statistics. Forensic Sci Int 2001;115:15-32.
24. Furu K, Wettermark B, Andersen M, et al. The Nordic countries as a cohort for pharmacoepidemiological research. Basic Clin Pharmacol Toxicol 2010;106:86-94.

25. Armitage JN, van der Meulen JH. Royal College of Surgeons Comorbidity Consensus Group. Identifying co-morbidity in surgical patients using administrative data with the Royal College of Surgeons Charlson Score. Br J Surg 2010;97:772-81.

26. Cutler SJ, Ederer F. Maximum utilization of the life table method in analyzing survival. J Chronic Dis 1958;8:699-712.

27. Rice TW, Rusch VW, Apperson-Hansen C, et al. Worldwide esophageal cancer collaboration. Dis Esophagus 2009;22:1-8.

28. Low DE, Alderson D, Cecconello I, et al. International Consensus on Standardization of Data Collection for Complications Associated With Esophagectomy: Esophagectomy Complications Consensus Group (ECCG). Ann Surg 2015;262:286-94. 